\title{
Drug concentration in lung tissue in multidrug-resistant tuberculosis
}

\author{
To the Editor:
}

Multidrug-resistant tuberculosis (MDR-TB) is emerging worldwide, with 3.7\% of new cases and $20 \%$ of previously treated tuberculosis (TB) cases having MDR-TB. Unfortunately, second-line TB drugs, used for MDR-TB treatment, are less effective than first-line drugs [1]. Sputum culture rather than sputum smear microscopy is recommended to monitor treatment response [1]. Therapeutic drug monitoring (TDM), which may help optimise efficacy and minimise side-effects with the potential to safeguard intestinal absorption of drugs [2], is currently not recommended in World Health Organization (WHO) treatment guidelines [1]. Although TDM yields information on serum drug concentrations, penetration of second-line TB drugs into diseased tissues such as destroyed lung tissue has, to our knowledge, not been addressed in studies [3].

Herein, we report simultaneous blood and tissue concentrations of second-line TB drugs in lung tissue destroyed by MDR-TB.

A 13-year-old Somalian, HIV sero-negative female, residing in the Netherlands since 2010, was admitted to our TB Unit (TB Center Beatrixoord, University Medical Center Groningen, Groningen, the Netherlands) in September 2011 with a 3-month history of cough, fever, chest pain and unintentional $14 \mathrm{~kg}$ weight loss. TB contacts were denied and she had not received a bacilli Calmette-Guérin vaccination. Apart from almost absent breathe sounds and dullness to percussion over the left lung field her physical examination was normal.

Chest radiography showed infiltrates in the left lung, particularly in the left upper lobe. Sputum microscopy revealed acid-fast bacilli, and she was started on TB treatment with isoniazid, rifampicin, pyrazinamide and ethambutol. 2 weeks later, molecular diagnostic tests revealed mutations in both the kat $G$ and $r p o B$ genes. MDR-TB was now considered and she was transferred to our hospital (University Medical Center Groningen).

We changed her treatment to pyrazinamide, ethambutol, kanamycin and moxifloxacin [4]. Drug susceptibility testing (DST) by the National Tuberculosis Reference Laboratory showed resistance to all first-line drugs, as well as protionamide. The isolate appeared susceptible to co-amoxiclav, amikacin, capreomycin, moxifloxacin and linezolid, as well as ertapenem and co-trimoxazole [5]. We started her on linezolid and ertapenem to replace ethambutol and pyrazinamide. Trough drug concentrations, part of $\mathrm{TDM}$, as routinely performed in our centre, were $0.5 \mathrm{mg} \cdot \mathrm{L}^{-1}$ and $0.5 \mathrm{mg} \cdot \mathrm{L}^{-1}$ for linezolid and moxifloxacin, respectively. The minimal inhibitory concentrations for linezolid and moxifloxacin were $0.25 \mathrm{mg} \cdot \mathrm{L}^{-1}$ and $0.125 \mathrm{mg} \cdot \mathrm{L}^{-1}$, respectively (D. van Soolingen, National Tuberculosis Reference Laboratory, National Institute for Public Health and the Environment (RIVM), Bilthoven, the Netherlands; personal communication). The area under the concentration-time curve (AUC) 0-24 for linezolid and moxifloxacin were $74.90 \mathrm{mg} \cdot \mathrm{h}^{-1} \cdot \mathrm{L}^{-1}$ and $30.04 \mathrm{mg} \cdot \mathrm{h}^{-1} \cdot \mathrm{L}^{-1}$, respectively. As these AUCs were adequate, no dose adjustment of either drug was performed.

The first sputum microscopy was negative after 6 weeks, and the sputum culture turned negative after 2 months. Afterwards, culture was positive on a further two occasions; at 3 and 5 months after starting treatment. No radiological improvement occurred, with atelectasis of the left upper lobe. We were concerned that the drugs would not penetrate into the affected lung; therefore, surgery was now considered. ${ }^{99} \mathrm{~m}$ Tc-macroaggregated albumin lung perfusion imaging (fig. 1), lung function testing and a computed tomography scan of the chest were performed to evaluate the relative contribution of the left lung for perfusion, and to assess her pulmonary reserve. It was concluded that the left lung hardly contributed to her pulmonary function and a left pneumonectomy was performed.

Immediately following surgery, samples of the left upper and left lower lobes were collected for microbiology and Prausnitz-Küstner testing. The left upper lobe was most affected and the left lower lung was less affected (fig. 1). Surgical specimens were all smear and culture negative. Samples from the left upper lobe tested positive by IS6110 PCR, and samples of the left lower lobe remained negative. The drug concentration of moxifloxacin was $0.73 \mu \mathrm{g}^{-1} \cdot \mathrm{g}^{-1}$ in the left upper lobe (most affected by TB), and the linezolid concentration was $3.87 \mu \mathrm{g}^{-1} \cdot \mathrm{g}^{-1}$. The concentrations in the left lower lobe (less affected) were 


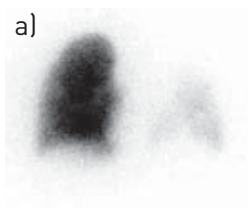

d)

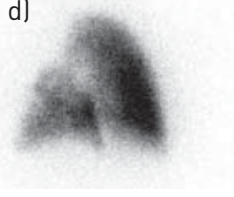

b)

e)

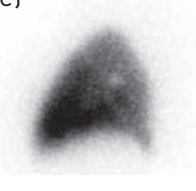

c)

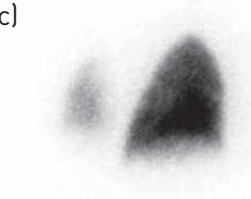

f)

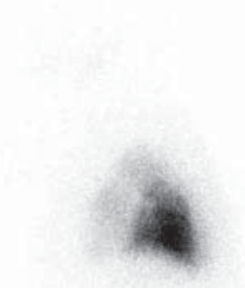

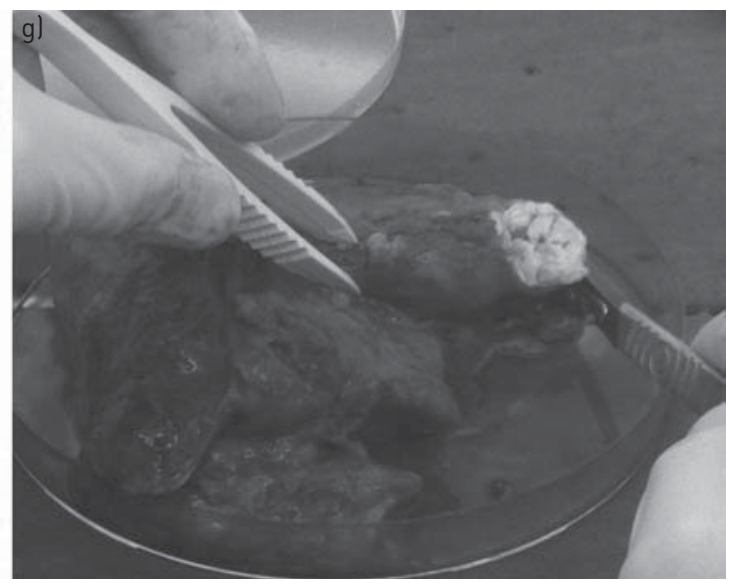

FIGURE 1 Lung perfusion scan with $72 \mathrm{MBq}{ }^{99 \mathrm{~m}} \mathrm{Tc}$-macroaggregated albumin 7.5 months after the start of treatment: a) anterior, b) posterior, c) right posterior oblique, d) left posterior oblique, e) right lateral and f) left lateral views. g) A photo of the resected lung. The left upper lobe is the lobe most affected by tuberculosis. The left/right perfusion percentage ( $90 \%$ versus $10 \%)$ was calculated from the geometric mean of the counts from both lungs in the anterior and posterior views, while the left upper/left lower lobe percentage (9\% versus $91 \%$ ) was calculated from the left posterior oblique image.

$0.95 \mu \mathrm{g}^{-1} \cdot \mathrm{g}^{-1}$ for moxifloxacin and $3.1 \mu \mathrm{g}^{-1} \cdot \mathrm{g}^{-1}$ for linezolid. At the time of pulmonary resection, $36 \mathrm{~h}$ had lapsed after the last oral drug dosage.

Consecutive chest radiographs showed gradual fluid filling of the pleural cavity. Our patient was discharged 2 months after pneumonectomy in a good condition. Her TB treatment regimen was switched to an oral regimen, consisting of co-trimoxazole, moxifloxacin and clofazimine (five times per week, $100 \mathrm{mg}$ ) for another 10 months. She has been well, and at the time of writing this report, 4 months before completion of drug therapy, she reported being well with no signs of relapse and no side-effects with improved exercise tolerance.

For the first time, we describe blood and tissue concentrations of second-line TB drugs. We showed that drug penetration was excellent, with similar drug concentrations in severely TB-affected lung tissue and in less affected lung tissue. Only one report, an overview by DARTOIs et al. [3], has addressed the question of how well second-line TB drugs penetrate into diseased lung and other tissues in relation to TDM but, unlike data on first-line drugs in resected lung tissue, no paired tissue and blood data have been reported on second-line drugs to date. Only data from rabbit models showed that moxifloxacin had a better AUC in lung and lesion tissue relative to plasma AUC compared to the first-line TB drugs [6].

Tailored regimens for MDR-TB treatment can be started after DST results are known. Efficacy of treatment for MDR-TB is solely based on sputum smear and culture status [1]. Studies with individualised treatment that studied treatment success have predominantly been based on DST results [7], without using pharmacokinetics with TDM. Although TDM may help evaluate TB drug absorption, even this aspect has not been extensively studied or practiced [2]. Therefore, to date, the full potential of TDM and drug penetration into TB-affected tissues has not been studied.

Surgery added to chemotherapy for MDR-TB has been described in case series but no randomised controlled trials have been conducted to evaluate this approach. In reported series, selection bias by indication is a potential flaw as patients who underwent surgery were perhaps younger or in a better clinical condition. However, even with these limitations, surgical resection has been suggested early in the course of treatment of MDR-TB $[8,9]$. A recent meta-analysis showed that the treatment effect of surgery added to chemotherapy was more pronounced in studies with extensively drug-resistant TB. This meta-analysis also showed that the treatment effect was stronger in MDR-TB studies with isolates showing resistance to more than 4.7 drugs [10].

Surgery added to chemotherapy was widely used in the pre-rifampicin era, even at our centre as shown in the thesis of MULDER-DE JONG [11], with 25\% of samples being culture positive and 96\% positive on microscopy. A limitation in our approach was that we took only one sample per resected lobe.

In our patient a pneumonectomy was performed because we feared that drugs would not reach the most severely affected parts of the destroyed lung, potentially resulting in persistent organisms that might subsequently cause a relapse or treatment failure if sub-therapeutic drug concentrations resulted in additional drug resistance. With a 36-h delay between the last dose of moxifloxacin and linezolid before surgery, both drugs were still detectable equally well. We have shown, although just in one patient, that treatment regimens yielding adequate blood concentrations as evidenced by TDM may provide similarly 
adequate penetration in affected tissue; non-resolving pulmonary infiltrates in TB patients do not necessarily preclude inadequate drug penetration.

。 @ERSpublications

Treatment regimens yielding adequate blood concentrations may provide similarly adequate penetration in affected tissue http://ow.ly/p7f9F

Onno W. Akkerman ${ }^{1}$, Richard van Altena ${ }^{1}$, Theo Klinkenberg ${ }^{2}$, Adrienne H. Brouwers ${ }^{3}$, Alphons H.H. Bongaerts ${ }^{4}$, Tjip S. van der Werf ${ }^{1,5}$ and Jan-Willem Alffenaar ${ }^{6}$

${ }^{1}$ Dept of Pulmonology and Tuberculosis, University of Groningen, University Medical Center Groningen, Groningen,

${ }^{2}$ Dept of Cardiothoracic Surgery, University of Groningen, University Medical Center Groningen, Groningen, ${ }^{3}$ Dept of Nuclear Medicine and Molecular Imaging, University of Groningen, University Medical Center Groningen, Groningen, ${ }^{4}$ Dept of Radiology, University of Groningen, University Medical Center Groningen, Groningen, ${ }^{5}$ Dept of Internal Medicine, University of Groningen, University Medical Center Groningen, Groningen, and ${ }^{6}$ Dept of Hospital and Clinical Pharmacy, University of Groningen, University Medical Center Groningen, Groningen, The Netherlands.

Correspondence: O.W. Akkerman, Dept of Pulmonology and Tuberculosis (AA11), University of Groningen, University Medical Center Groningen, P.O. Box 30.001, 9700 RB Groningen, The Netherlands. E-mail: o.w.akkerman@umcg.nl

For editorial comments see page 1449 .

Received: March 172013 | Accepted after revision: April 102013

Conflict of interest: Disclosures can be found alongside the online version of this article at www.erj.ersjournals.com

\section{References}

1 Falzon D, Jaramillo E, Schunemann HJ, et al. WHO guidelines for the programmatic management of drug-resistant tuberculosis: 2011 update. Eur Respir J 2011; 38: 516-528.

2 Bolhuis MS, Altena RV, Soolingen DV, et al. Clarithromycin increases linezolid exposure in multidrug-resistant tuberculosis patients. Eur Respir J 2013 [in press DOI: 10.1183/09031936.00001913].

3 Dartois V, Barry CE. Clinical pharmacology and lesion penetrating properties of second- and third-line antituberculous agents used in the management of multidrug-resistant (MDR) and extensively-drug resistant (XDR) tuberculosis. Curr Clin Pharmacol 2010; 5: 96-114.

4 Pranger AD, van Altena R, Aarnoutse RE, et al. Evaluation of moxifloxacin for the treatment of tuberculosis: 3 years of experience. Eur Respir J 2011; 38: 888-894.

5 Alsaad N, van Altena R, Pranger AD, et al. Evaluation of co-trimoxazole in treatment of multidrug-resistant tuberculosis. Eur Respir J 2013; 42: 504-512.

6 Kjellsson MC, Via LE, Goh A, et al. Pharmacokinetic evaluation of the penetration of antituberculosis agents in rabbit pulmonary lesions. Antimicrob Agents Chemother 2012; 56: 446-457.

7 Orenstein EW, Basu S, Shah NS, et al. Treatment outcomes among patients with multidrug-resistant tuberculosis: systematic review and meta-analysis. Lancet Infect Dis 2009; 9: 153-161.

8 Iseman MD, Madsen L, Goble M, et al. Surgical intervention in the treatment of pulmonary disease caused by drugresistant Mycobacterium tuberculosis. Am Rev Respir Dis 1990; 141: 623-625.

9 Kempker RR, Vashakidze S, Solomonia N, et al. Surgical treatment of drug-resistant tuberculosis. Lancet Infect Dis 2012; 12: 157-166.

10 Marrone MT, Venkataramanan V, Goodman M, et al. Surgical interventions for drug-resistant tuberculosis: A systematic review and meta-analysis. Int J Tuberc Lung Dis 2013; 17: 6-16.

11 M.T. Mulder-de Jong. Over de tuberkelbacteriën in gereseceerde longdelen [About the tubercle bacilli in resected lung sections]. PhD thesis. University of Groningen, Groningen, the Netherlands, 1960.

\section{Neutrophilia independently predicts death in tuberculosis}

To the Editor:

Experimental animal work indicates that neutrophils play a key role in the immune response to mycobacteria [1, 2]. They appear protective against early infection [3] but in established disease, neutrophilia associates with pathology $[1,4]$. In humans, higher neutrophil counts at tuberculosis diagnosis predict slower sputum conversion to negative during therapy $[5,6]$, but the overall prognostic significance of neutrophilia in human tuberculosis remains elusive. We therefore aimed to analyse this phenomenon in a study powered to detect an independent relationship with mortality. 